\title{
Contextos educativos como área de participación: de la legalidad a la innovación
}

Educational contexts as an area of participation: from legality to innovation

\author{
Mónica Guerra Santana \\ Profesora Ayudante Doctora \\ Departamento de Educación \\ Universidad de Las Palmas de Gran Canaria (España) \\ E-mail: monica.guerra@ulpgc.es \\ Josefa Rodríguez Pulido \\ Profesora Titular de Universidad \\ Departamento de Educación \\ Universidad de Las Palmas de Gran Canaria (España) \\ E-mail: josefa.rodríguez@ulpgc.es \\ Josué Artiles Rodríguez \\ Profesor Contratado Doctor \\ Departamento de Educación \\ Universidad de Las Palmas de Gran Canaria (España) \\ E-mail: josue.artiles@ulpgc.es
}

Resumen: El centro educativo debe de estar al servicio de las familias, de ahí que,
en contextos de desventaja social, las escuelas públicas deben coordinarse con otras
organizaciones de la comunidad para ofertar sus espacios para el bien común. En la
actualidad en España contamos con un marco institucional que apoya y reafirma la
participación de las familias en los centros educativos de manera formal, pero en
cambio, al parecer, la realidad es bien distinta. La existencia de una escasa
implicación de los padres y las madres en los órganos de participación en los centros
educativos es evidenciada por numerosos estudios realizados. El presente trabajo 
tiene como objetivo abordar la importancia de la participación familiar en la institución educativa y cómo la coordinación y el apoyo entre ambos permitirá el pleno desarrollo social, psicológico y educativo del alumnado. Para lo cual la ley educativa establece sus protocolos pertinentes, de ahí que pretendemos presentar un estudio de caso concreto basado en las experiencias de participación familia-escuela donde, además, la figura del educador social juega un rol de gran importancia al incorporar a estos profesionales en la escuela. Con esta medida, pretenden conseguir el pleno desarrollo personal de cada estudiante, destacando principalmente la formación en valores, la resolución de conflictos, el control del absentismo escolar, la prevención del acoso escolar o el desarrollo de una convivencia cívica y democrática.

Palabras clave: Familia; Instituciones educativas; Participación familiar; Educador social; Innovación.

\begin{abstract}
The educational center must be at the service of families, which is why, in contexts of social disadvantage, public schools must coordinate with other community organizations to offer their spaces for the common good. Currently in Spain we have an institutional framework that supports and reaffirms the participation of families in schools in a formal way, but instead, apparently, the reality is quite different. The existence of a low involvement of fathers and mothers in the participation bodies in educational centers is evidenced by numerous studies. The present work aims to address the importance of family participation in the educational institution and how the coordination and support between the two will allow the full social, psychological and educational development of the students. For which the educational law establishes its relevant protocols, hence we intend to present a concrete case study based on the experiences of family-school participation where, in addition, the figure of the social educator plays a role of great importance when incorporating these professionals at school. With this measure, they aim to achieve the full personal development of each student, mainly highlighting the
\end{abstract}


formation in values, the resolution of conflicts, the control of truancy, the prevention of bullying or the development of a civic and democratic coexistence.

Keywords: Family; Educational institutions; Family involvement; Social educator; Innovation.

\section{Instituciones educativas y familias}

La familia es una institución universal presente en todas las sociedades. Cuando hablamos de familia la entendemos como la unión de diferentes personas que comparten un proyecto vital común, donde se generan sentimientos de pertenencia y con un compromiso social entre sus miembros de reciprocidad (Rodrigo y Palacios, 1998). Al pensar en la familia como institución, la entendemos como un pilar básico social donde confluyen los procesos de convivencia, afecto, cuidado, etc., que ha sufrido en los últimos años múltiples cambios tanto en su estructura como en su funcionamiento, debido en parte, a los profundos cambios sociales, económicos y políticos acaecidos (Godoy, 2018). La familia como institución social, evoluciona en el tiempo y en el contexto donde se desarrolla adaptándose a las circunstancias del momento. Se trata de una institución universal, donde una gran cantidad de organizadores sociales condicionan la vida en familia como, por ejemplo, la cultura, la religión, la política, las ideologías, etc. (Benítez Pérez, 2017).

Nadie pone en duda que la familia es el principal agente socializador del niño, y junto a ella está la escuela. La familia como institución socializadora favorece tanto el desarrollo emocional de los hijos e hijas asegurando de esta manera su identidad personal y social (Huayamave, Benavides y Sangacha, 2019). Ortiz Cermeño (2011) evidencia que ambas deben complementarse con el único fin de satisfacer las necesidades formativas y sociales de las personas, de ahí que el sistema educativo debe contar con la colaboración y el apoyo de las familias. Los centros educativos se crearon con el fin de favorecer el desarrollo de los menores y apoyar a los padres y madres en su labor educativa, por lo tanto, resulta evidente la colaboración entre 
ambas (Sosa, 2009). Esta necesidad ya la recogía la Ley General de Educación de 1970, en su artículo 5.2:

\footnotetext{
"La familia tiene como deber y derecho primero e inalienable la educación de sus hijos. En consecuencia, constituye una obligación familiar, jurídicamente exigible, cumplir y hacer cumplir las normas establecidas en materia de educación obligatoria, ayudar a los hijos a beneficiarse de las oportunidades que se les brinden para estudios posteriores y coadyuvar a la acción de los centros docentes".
}

Por lo tanto, familia y escuela deben caminar juntas hacia un mismo fin común, que es el desarrollo global de los niños. No olvidemos que tal y como nos dice San Fabián (2006), una de las primeras decisiones que toma la familia es la elección del centro educativo, lo que implica conocer si el proyecto educativo se asemeja a sus valores e intereses como familia.

Hoy en día escuchamos voces que afirman que las familias delegan sus funciones en la escuela, y, por otro lado, que la escuela no permite la participación de las familias por miedo o inseguridad (Segovia, Titos y Martos, 2016). Muchos padres y madres reconocen la importancia de participar de manera activa en la escuela, pero a veces no saben cómo hacerlo o simplemente no quieren (Entrena y Soriano, 2003). Esta situación debe cambiar si como sociedad queremos participar en los retos que nos plantea el futuro. Por lo tanto, las familias deben conocer y ser conscientes de la responsabilidad que tienen sobre la educación de sus hijos y las escuelas tienen que fomentar la participación de los padres y las madres en el centro educativo (Mestres, 2012). La suma de esfuerzos entre estas dos instituciones, familia y escuela, deber ser el camino para la consecución de una formación integral y completa del niño (Maestre, 2009). Pero siempre teniendo en cuenta, que en esta suma debe existir el equilibrio, las relaciones entre la institución educativa y los padres y las madres debe ser recíproca. Las familias no deben sentirse clientes de las escuelas, solo considerando la relación entre ellos en torno a las calificaciones académicas, porque de esta manera es muy probable que se genere desconfianza, reproches y actitudes negativas nada favorables en el desarrollo del menor. En muchas ocasiones, el grado de compromiso e implicación que asumen las familias depende tanto de la actitud 
que éstas tengan hacia la participación, pero también de la disposición que manifiesten los maestros (Cárcamo-Vásquez y Rodríguez-Garcés, 2015). Según el Informe Europeo sobre la Calidad de la Educación Escolar, una actitud crítica y exigente por parte de los padres y las madres puede lograr o bien impedir una óptima relación familia-escuela. Si las familias adoptan un rol crítico pero constructivo ayudarán a la mejora de los centros gracias a su apoyo a la dirección y al profesorado, pero, en cambio, si la crítica es destructiva puede generar conflictos en las relaciones entre ambos (Comisión Europea, 2000).

Son múltiples las definiciones del concepto de familia, coincidiendo en la relación intensa que se produce entre sus miembros resultando un factor protector de sus miembros que favorece la construcción de valores y la implantación de normas que permiten que se creen oportunidades favorecedoras de las propias experiencias (Marín, Quintero y Rivera Gómez, 2019). Las sociedades democráticas del siglo XXI destacan por su pluralidad y la complejidad de sus estructuras y tipos de familia (Pliego, 2013). La familia se debe entender como un sistema complejo compuesto a su vez por diferentes subsistemas abiertos y expuestos a diferentes transformaciones (Adolfi, 1991). Lejos queda el modelo de familia tradicional, compuesto por un hombre y una mujer y los hijos tenidos en común que conviven en el mismo hogar. La situación actual es diferente, la familia como institución se muestra como un sistema abierto con múltiples posibilidades y estructuras que dan lugar a diversos tipos de familia. Ahora los miembros de la familia se relacionan o vinculan por lazos de afecto y respeto (Oliva, 2013). Como grupo social ha modificado tanto su estructura como sus modelos, adaptándose a las nuevas dinámicas y costumbres que marca la sociedad actual. Como afirma Engels (2008), la familia es un elemento activo que evoluciona con la sociedad, es el núcleo donde se comparten y articulan los riesgos sociales de sus miembros (Carbonell, Carbonell y González Martín, 2012). 
Los tipos de familias que conviven en la actualidad son (Martín y Mesa, 2015):

- Familias Biparentales: formadas por el padre, la madre y los hijos de ambos que conviven juntos. Hablamos de familias biparentales nucleares, cuando sus miembros conforman un núcleo familiar; y, biparentales polinucleares, si éstos se unen a otros núcleos familiares extensos.

- Familias Monoparentales: donde un progenitor es el responsable de sus hijos menores que conviven con él o ella. Si constituyen un solo núcleo familiar hablamos de familias monoparentales nucleares, y si esta se encuentra dentro de una familia compleja, por ejemplo, la formada por los abuelos de los niños, hablamos de familia monoparental polinuclear.

- Familias Adoptivas: formada por la adopción de uno o varios niños por parte de personas que no tienen relación biológica. Hoy en día, encontramos familias adoptivas que adoptan el papel de familias tanto biparentales como monoparentales.

- Familias Reconstituidas o simultáneas: formadas por personas que, tras una ruptura, reinician sus vidas en familia con un nuevo cónyuge. Según Visher y Visher (1988), hablamos de familias reconstituidas cuando al menos uno de los cónyuges aporta un hijo de otra relación.

- Familias Homoparentales: formadas por la unión de dos personas del mismo género, donde uno de los progenitores o ambos contribuye con los hijos tenidos en relaciones heterosexuales, o bien deciden iniciar el proceso de adopción, o a través de las técnicas de reproducción asistida.

Quintero (2007), complementa esta tipología añadiendo otras estructuras familiares en las que entran en juego otros factores como:

- Familias biculturales o multiculturales (transnacionales). Formadas por matrimonios o parejas mixtas, donde la primera nacionalidad o cultura de uno de sus miembros no es la española. También hablamos de familias multiculturales cuando 
uno de los progenitores pertenece a otra cultura, bien por adopción o bien por una unión anterior.

- Familia mixta simple. Se trata de la familia nuclear que ha tenido que marcharse de su país de manera forzada y se ha unido a otra tipología familiar; por ejemplo, la unión obligada de dos familias para poder sobrevivir.

- Familia mixta compleja. Sigue el modelo anterior, pero la unión es de más de dos familias, donde la estructura, el funcionamiento y el ciclo vital son distintos entre ellas.

Existen múltiples estructuras familiares, pero sus funciones como familia son las mismas. Tienen que garantizar la nutrición, la educación, la estabilidad emocional y afectiva, la asistencia a los servicios de salud y, un óptimo desarrollo de la autoestima de sus miembros. Si seguimos el enfoque evolutivo-educativo de Rodrigo, Máiquez, Martín y Byrne (2008) los padres y madres tienen la función de fomentar el desarrollo y el aprendizaje de sus hijos, guiándolos y orientándoles en su desarrollo. Algunas funciones que deben desarrollar las familias son: la perpetuación de su identidad social; el reconocimiento y el respeto de la autoridad; educar en diálogo y resolución de conflicto; el respeto a las normas de convivencia, entre otras (Oliva, 2013). La familia debe educar a sus hijos con el objetivo de dotarlos de herramientas, estrategias y recursos que les preparen para su trayecto vital. De ahí que autores como Moreno-Acero, Leyva-Townsend, y Parra-Moreno (2019), vean a la familia como el primer entorno educativo encargado de formar en valores cívicos a sus miembros. Martínez (2005) identifica siete valores que conforman el actual modelo social de la familia: la libertad de los miembros de la pareja a la hora de tomar decisiones; el bienestar, alejándonos del modelo de sacrificio de las familias tradicionales; la igualdad, donde ambos cónyuges tienen los mismos derechos; solidaridad entre todos los miembros que constituyen la familia; la tolerancia a la diversidad; el individualismo y la privacidad; y, por último, la búsqueda de la felicidad por los miembros de la familia. 
Por lo tanto, la familia al igual que la escuela constituye una institución y, es por eso, que debe analizarse y comprenderse como tal, con sus reglas, estructuras organizativas, modelos de comunicación, etc.

\section{La Escuela como un espacio de participación familiar}

La Constitución Española (1978), en su artículo 27, ${ }^{1}$ hace referencia a la libertad de enseñanza, el derecho a la educación y a la autonomía universitaria, de esta manera reconoce, entre otros, el derecho de todas las personas a recibir educación bajo el principio de la libertad de enseñanza, marca como objeto de la educación el desarrollo de la personalidad humana siempre respetando los principios democráticos y libertades fundamentales, siendo los poderes públicos, quienes deban garantizar el derecho de todos a la educación, además, en dicho artículo evidencia que tanto los docentes como los padres y los estudiantes intervendrán en el control y gestión de los centros educativos. Por lo tanto, no nos puede sorprender que las diferentes leyes educativas españolas recojan estos principios, enfatizando el derecho de todos y todas a recibir una educación de calidad bajo los principios de equidad e igualdad de oportunidades, donde poco a poco se ha ido definiendo el papel de los padres y las madres en la colaboración con los centros escolares. Para Espejo Yasik (2014), la garantía del derecho a la educación debe estar en la relación entre una

${ }^{1}$ Constitución Española. «BOE» núm. 311, de 29 de diciembre de 1978. Referencia: BOE-A-197831229.

Artículo 27:

1. Todos tienen el derecho a la educación. Se reconoce la libertad de enseñanza.

2. La educación tendrá por objeto el pleno desarrollo de la personalidad humana en el respeto a los principios democráticos de convivencia y a los derechos y libertades fundamentales.

3. Los poderes públicos garantizan el derecho que asiste a los padres para que sus hijos reciban la formación religiosa y moral que esté de acuerdo con sus propias convicciones.

4. La enseñanza básica es obligatoria y gratuita.

5. Los poderes públicos garantizan el derecho de todos a la educación, mediante una programación general de la enseñanza, con participación efectiva de todos los sectores afectados y la creación de centros docentes.

6. Se reconoce a las personas físicas y jurídicas la libertad de creación de centros docentes, dentro del respeto a los principios constitucionales.

7. Los profesores, los padres y, en su caso, los alumnos intervendrán en el control y gestión de todos los centros sostenidos por la Administración con fondos públicos, en los términos que la ley establezca. 8. Los poderes públicos inspeccionarán y homologarán el sistema educativo para garantizar el cumplimiento de las leyes.

9. Los poderes públicos ayudarán a los centros docentes que reúnan los requisitos que la ley establezca. 10. Se reconoce la autonomía de las Universidades, en los términos que la ley establezca. 
educación de calidad que garantice el principio de igualdad de oportunidades y el desarrollo de una vida independiente. En sociedades donde existe una brecha de desigualdad entre sus ciudadanos, el derecho a la educación garantiza las políticas de cohesión social e igualdad de oportunidades.

El derecho a la educación es universal, tal y como se recoge en la Declaración Universal de los Derechos Humanos (1948), en su artículo 26 mostrando el derecho de todas las personas a recibir educación, ${ }^{2}$ con el objetivo de desarrollar la personalidad humana, además, del derecho de los padres a elegir el tipo de educación de sus hijos e hijas. Una educación de calidad revierte en ciudadanos que participan ejerciendo sus derechos de manera responsable en democracia (Castellanos, 2019).

A lo largo de los años la institución educativa ha ido abriendo sus puertas a la participación de las familias, en parte por la imposición dada por la legislación española. En un primer momento, es la Ley Orgánica General de Educación (LGE) la que en 1970 refleja la necesidad de que la familia participe en los centros educativos. Con la Ley Orgánica del Estatuto de Centros Escolares (LOECE) ${ }^{3}$ en 1980 el Consejo de Dirección y la Junta Económica se crean como nuevos órganos de participación para las familias. Es en 1985 con la Ley Orgánica del Derecho a la Educación (LODE) ${ }^{4}$ cuando se da más libertad para crear las asociaciones de padres, a la vez que se aumentaron las funciones del consejo escolar. Con la Ley Orgánica

\footnotetext{
2 Declaración Universal de los Derechos Humanos. Asamblea General de la ONU, 10 de diciembre de 1948, París.

Artículo 26.

1. Toda persona tiene derecho a la educación. La educación debe ser gratuita, al menos en lo concerniente a la instrucción elemental y fundamental. La instrucción elemental será obligatoria. La instrucción técnica y profesional habrá de ser generalizada; el acceso a los estudios superiores será igual para todos, en función de los méritos respectivos.

2. La educación tendrá por objeto el pleno desarrollo de la personalidad humana y el fortalecimiento del respeto a los derechos humanos y a las libertades fundamentales; favorecerá la comprensión, la tolerancia y la amistad entre todas las naciones y todos los grupos étnicos o religiosos, y promoverá el desarrollo de las actividades de las Naciones Unidas para el mantenimiento de la paz.

3. Los padres tendrán derecho preferente a escoger el tipo de educación que habrá de darse a sus hijos.

${ }^{3}$ Ley Orgánica 5/1980, de 19 de junio, por la que se regula el Estatuto de Centros Escolares. «BOE» núm. 154, de 27 de junio de 1980. Referencia: BOE-A-1980-13661.

${ }^{4}$ Ley Orgánica 8/1985, de 3 de julio, reguladora del Derecho a la Educación. «BOE» núm. 159, de 4 de julio de 1985. Referencia: BOE-A-1985-12978.
} 
de Ordenación General del Sistema Educativo (LOGSE) ${ }^{5}$ en 1990, se hace hincapié en la importancia de la participación familiar para lograr los objetivos educativos. En el 2002, la Ley Orgánica de Calidad de la Enseñanza (LOCE) ${ }^{6}$ en su artículo 3.1.c. limita la participación de las familias a valorar la evolución de la educación de sus hijos e hijas. Es la Ley Orgánica de Educación $(\mathrm{LOE})^{7}$, la que en 2006 refuerza la importancia de la participación de la familia en la escuela. Destacar la indicación que realiza en la colaboración y el compromiso de los padres con los centros docentes. La Ley Orgánica para la Mejora de la Calidad Educativa (LOMCE) ${ }^{8}$ de 2013 refuerza lo dicho por la LOE, pero añadiendo en su artículo 132 que se debe impulsar la colaboración con las familias o cualquier institución que facilite la relación del centro con el entorno social de los niños entre otros (Tabla 1).

Tabla 1. Evolución y Aportaciones de las Leyes Educativas a la participación familiar

\begin{tabular}{|c|c|}
\hline \multicolumn{2}{|c|}{$\begin{array}{l}\text { LEYES EDUCATIVAS APORTACIONES A LA PARTICIPACIÓN } \\
\text { FAMILIAR }\end{array}$} \\
\hline LGE (1970) & $\begin{array}{l}\text { Recoge la necesidad de que las } \\
\text { familias participen en los } \\
\text { centros escolares }\end{array}$ \\
\hline LOECE (1980) & $\begin{array}{l}\text { Crea el Consejo de Dirección y } \\
\text { la Junta Económica como } \\
\text { nuevos órganos para fomentar } \\
\text { la participación familiar }\end{array}$ \\
\hline LODE (1985) & $\begin{array}{l}\text { Concede mayor libertad para la } \\
\text { creación de las asociaciones de }\end{array}$ \\
\hline
\end{tabular}

\footnotetext{
${ }^{5}$ Ley Orgánica 1/1990, de 3 de octubre, de Ordenación General del Sistema Educativo. «BOE» núm. 238, de 4 de octubre de 1990. Referencia: BOE-A-1990-24172.

${ }^{6}$ Ley Orgánica 10/2002, de 23 de diciembre, de Calidad de la Educación. «BOE» núm. 307, de 24 de diciembre de 2002. Referencia: BOE-A-2002-25037.

${ }^{7}$ Ley Orgánica 2/2006, de 3 de mayo, de Educación. «BOE» núm. 106, de 4 de mayo de 2006. Referencia: BOE-A-2006-7899.

${ }^{8}$ Ley Orgánica 8/2013, de 9 de diciembre, para la mejora de la calidad educativa. «BOE» núm. 295, de 10 de diciembre de 2013. Referencia: BOE-A-2013-12886.
} 


\begin{tabular}{|c|c|}
\hline & $\begin{array}{l}\text { padres y aumentan las } \\
\text { funciones del consejo escolar }\end{array}$ \\
\hline LOGSE (1990) & $\begin{array}{l}\text { Refuerza la importancia de la } \\
\text { participación familiar para el } \\
\text { logro de los objetivos } \\
\text { educativos }\end{array}$ \\
\hline LOCE (2002) & $\begin{array}{l}\text { Limita la participación de las } \\
\text { familias a la valoración de la } \\
\text { evolución de la educación de } \\
\text { sus hijos }\end{array}$ \\
\hline LOE (2006) & $\begin{array}{l}\text { Refuerza la colaboración y el } \\
\text { compromiso de los padres con } \\
\text { los centros docentes } \\
\text { fomentando la interacción } \\
\text { familia-escuela }\end{array}$ \\
\hline LOMCE (2013) & $\begin{array}{l}\text { Impulsa la colaboración con las } \\
\text { familias que facilite la relación } \\
\text { del centro con el entorno social } \\
\text { de los estudiantes }\end{array}$ \\
\hline
\end{tabular}

Fuente: Elaboración Propia

Por lo tanto, tenemos un marco institucional que apoya y reafirma la participación de las familias en los centros educativos de manera formal, pero, en cambio, son varios los estudios que evidencian la baja implicación de los padres y las madres en los órganos de participación colectiva: Consejos Escolares y Asociaciones de Madres y Padres (AMPAS) (Santos Guerra, 1997; San Fabián, 1997; Martín-Moreno, 2000 y Egido, 2014). Aún no se ha conseguido involucrar a las familias de manera real en las estructuras de participación escolar (Torres, 2013). Para Bolívar (2006), esta disminución de la participación de las familias en los Consejos Escolares nos debe llevar a plantearnos nuevas maneras de implicar a los padres y madres en la comunidad educativa. Por ejemplo, Rego, Moledo y Caamaño (2019) proponen 
programas específicos de actuación desde los centros educativos que fomenten la participación de las familias. Si queremos mejorar e incrementar la participación familiar en las escuelas, los centros educativos tienen que diseñar actividades para tal fin, delimitando de manera clara los tipos de participación que se podrán realizar y el rol que tendrá cada familia durante este proceso (Calvo, Verdugo y Amor, 2016). Autores como Llevot y Bernad (2015), ven necesario que las AMPAS respondan a las necesidades de participación de las familias a través del diseño de estrategias y medios de actuación eficaces. Podrían responsabilizarse de actividades que ayuden a mejorar la cohesión social de la comunidad escolar, generando así un espacio educativo, por ejemplo, a través de las escuelas de padres y madres (Vilreales, Álvarez y Cerón, 2004). Pero no sólo en estos órganos, sino en general en la vida educativa de los centros, quizás se hace necesario proponer otras maneras de participación en los centros que se adapten a la realidad de las familias y al contexto social donde se ubican. Se puede lograr una educación óptima e integral de la persona cuando la escuela se interesa por conocer el medio en el que viven sus alumnos/as y abre sus puertas a la participación de las familias (Moya, 2018). En el Marco de Acción de la Declaración de Salamanca se alentó a las escuelas, a los docentes y a los propios padres para que aunaran lazos de cooperación entre ellos (UNESCO, 1994). Warren (2005), ya proponía distintos enfoques que propiciaban las interacciones entre la familia, el centro y la comunidad. El autor hablaba del enfoque Full-Service Model, de integración de los servicios comunitarios, dirigido a las familias de zonas desfavorecidas; el enfoque Family Involvement (funcional de implicación de las familias), el cual analiza los roles e implicaciones tanto de los profesores como el de las familias con el fin de desarrollar el aprendizaje de los estudiantes; y, el enfoque Organizativo, donde recoge la importancia de establecer relaciones e interacciones con la comunidad.

A mayor diálogo y comunicación entre familia y docentes mayor confianza, coordinación e implicación entre ambos, lo cual repercute directamente en el rendimiento del alumnado (Epstein, 2001) y en la mejora de la participación (Hernández, Viudez y Guerrero, 2015). 
Éstos mismos resultados los corroboran, por ejemplo, González-Pienda y Núñez (2005), cuando afirman que existe una relación directa entre la implicación de la familia en la escuela y el rendimiento académico de los alumnos. Por otro lado, García, Hernández, Galián y Belmonte (2020), refieren la importancia de la labor del docente a la hora de propiciar la participación familiar en los centros educativos. Muchos docentes asumen que tienen la responsabilidad de comunicarse con los padres y madres de su alumnado, desarrollando en muchos casos un papel de mediador y dinamizador entre ellos (Vicente, Prados, Sanz y Martínez, 2017). Aquellos docentes que tienen una buena competencia comunicativa tienen mayor probabilidad de asegurarse una participación efectiva de las familias en sus aulas y en el centro educativo. No basta con la intención de querer comunicarse con la familia del alumnado, sino que se tiene que saber cómo se debe comunicar para que esa interacción sea efectiva.

El centro educativo debe de estar al servicio de las familias, de ahí que, en contextos de desventaja social, las escuelas públicas deben coordinarse con otras organizaciones de la comunidad para ofertar sus espacios para el bien común. Por ejemplo, ampliando los horarios de los centros fuera de los horarios lectivos, organizando actividades culturales, escuelas de padres y madres, etc. En este contexto tienen mucha influencia las AMPAS, que surgen como un movimiento de padres disconformes con la situación de la época (Garreta, 2016). Estas participan activamente en desarrollar propuestas donde el centro escolar abra sus puertas a la comunidad educativa, y en especial a los padres y madres, tanto en horario escolar como extraescolar. La participación educativa obliga a la participación no sólo de las familias sino también del profesorado y de toda la comunidad donde convive el niño (Marina, 2004). Son varios los retos que aún deben conseguir las AMPAS, entre ellos (Vilreales, Álvarez y Cerón, 2004):

- $\quad$ El derecho a que todos los niños y niñas consigan una escolarización plena.

- El derecho a que se evalúe el aprendizaje a través de los profesos y no exclusivamente de los resultados. 
- $\quad$ El derecho a que las tareas escolares se adapten a las realidades de los niños.

- $\quad$ El derecho a que los estudiantes inmigrantes o el alumnado desfavorecido no constituyan un desequilibrio en las escuelas, sino que su distribución sea justa tanto en la red pública como concertada, favoreciendo así la educación multicultural.

- $\quad$ El derecho a que no se imparta ninguna ideología ni religión en los centros educativos.

\section{Experiencias de participación familia-escuela en la Comunidad Autónoma Canaria}

En este espacio se presentarán algunas de las propuestas que la Consejería de Educación, Cultura y Deporte del Gobierno de Canarias ha puesto en marcha para favorecer la apertura de los centros educativos a las familias. Dichos programas se encuentran publicados en la página web oficial de la Consejería de Educación y Universidades (2020) siendo visible para toda la comunidad educativa.

La Administración educativa canaria es consciente de cómo las situaciones familiares, personales, sociales, culturales o económicas de las familias influyen en el desarrollo óptimo del alumnado. Es por ello por lo que plantean diferentes medidas de apoyo a los centros educativos y a la comunidad educativa, entre ellas la aprobación de la Ley 6/2014, de 25 de julio, Canaria de Educación no Universitaria, ${ }^{9}$ donde se regula en el artículo 43 el Plan Estratégico de Atención a la Diversidad (PEAD), ${ }^{10}$ e incluye a los profesionales de la educación social para que intervengan en los centros educativos públicos canarios desde el curso 2017-2018. Los educadores sociales ya formaban parte de los centros de educación secundaria desde el curso 2002 en Extremadura, y en la Comunidad Autónoma de Castilla-La Mancha desde el 2003. Su función principal está relacionada con la mediación y la resolución

\footnotetext{
${ }^{9}$ «BOC» núm. 152, de 7 de agosto de 2014. Referencia: BOC-A-2014-152-3512. «BOE» núm. 238, de 1 de octubre de 2014. Referencia: BOE-A-2014-9901

${ }^{10}$ Artículo 43. Plan Estratégico de Atención a la Diversidad (PEAD). 1. Las medidas de atención a la diversidad adoptadas por cada centro deberán estar enmarcadas en el Plan Estratégico de Atención a la Diversidad (PEAD). La atención a la diversidad del alumnado recibirá una especial atención en el ordenamiento del sistema educativo canario.
} 
de conflictos, aunque poco a poco sus funciones se han ido ampliando (Galán y Castillo, 2008; Esteban, 2014). En educación primaria la Comunidad Autónoma Canaria es una de las pioneras al incorporar a estos profesionales en la escuela. Con esta medida, pretenden conseguir el pleno desarrollo personal de cada estudiante, destacando principalmente la formación en valores, la resolución de conflictos, el control del absentismo escolar, la prevención del acoso escolar o el desarrollo de una convivencia cívica y democrática. La incorporación del educador social en la escuela debe fomentar el crecimiento personal del alumnado, además del respeto y la tolerancia en la resolución de conflictos socioeducativos (Esteban, 2014). Se considera que el educador social actuará como guía de los miembros de la comunidad educativa, con la finalidad de lograr una adecuada participación e inclusión social entre sus miembros (Ortín, 2012). Para ello, el profesional de la educación social, como agente cualificado, tendrá que interactuar tanto con el alumnado, con el equipo docente, como con las familias. Con estas últimas, conformarán el enlace con el centro educativo de sus hijos. Tal y como establece la Ley del 6/2014, dentro de las funciones del educador social se encuentra la de potenciar las relaciones entre los estudiantes, la familia, el centro educativo y la comunidad donde se desarrollan; para ello, delimita las funciones de estos profesionales en seis puntos claves:

- $\quad$ Ayuda a la prevención del abandono y absentismo escolar.

- Acompañamiento a los estudiantes y sus familias que se encuentran en situaciones de riesgo psicosocial.

- $\quad$ Intervención activa con el alumnado y las familias de otras culturas.

- Facilitar por medio de estrategias de dinamización la participación de las familias y el entorno de la comunidad.

- Mantener a las familias informadas sobre las diferentes actuaciones que realizan los centros educativos.

- Mejorar y desarrollar las relaciones del centro educativo con el entorno social. 
Autores como Menacho (2013) justifican la actuación de los educadores sociales en los centros educativos por el cumplimento de las siguientes funciones, que como podremos observar no difieren en gran medida por las que hoy en día asume la Comunidad Autónoma Canaria:

- $\quad$ Ayuda en la elaboración de los documentos de organización y función de los centros educativos, especialmente en la detección de necesidades socioeducativas y en el análisis de entorno familiar.

- Labores de coordinación y apoyo al equipo docente en temas como: mediación, absentismo escolar, educación en valores, etc.

- $\quad$ Fomentar la participación familiar en las escuelas.

- $\quad$ Procurar la apertura y la coordinación de la escuela con otras instituciones del entorno social.

- Participar junto al departamento de orientación en los programas de convivencia de los centros.

- Elaborar, implementar y evaluar diferentes programas educativos que fomenten la educación integral del alumno y la convivencia en los centros.

Señalar que la Red Canaria de Centros para fomentar la participación es un programa de la Consejería de Educación, Cultura y Deportes del Gobierno de Canarias (2020), que tiene como principal objetivo posibilitar el trabajo conjunto y el intercambio de experiencias de participación entre los centros educativos y los agentes educativos. Entre los objetivos que proponen están:

- Desarrollar una red de centros educativos que integren como principal pilar la participación de la comunidad.

- $\quad$ Fomentar la participación educativa desde un proceso de mejora. 
- Dotar tanto a docentes, padres, madres y alumnos de estrategias que favorezcan la plena participación.

- Fomentar la participación educativa como una estrategia que permita el desarrollo de competencias o que promuevan actitudes y valores óptimos para la convivencia.

Con el fin de lograr estos objetivos, esta Red cuenta con diferentes programas destinados a las familias sobre participación educativa, con el principio de generar una escuela que dé respuesta a las necesidades que marca la sociedad actual. El principio por el que se rigen es atender a las demandas sociales actuales desde la responsabilidad y participación de todos los miembros implicados, profesores, familias y alumnos.

Para el buen ejercicio de la parentalidad y, por lo tanto, también la participación responsable de las familias en las escuelas, la Consejería de Educación, Cultura y Deportes del Gobierno de Canarias pone a disposición de las familias una plataforma on-line que les permite recibir formación tanto desde la modalidad en línea como presencial. Los cursos ofertados versan sobre diferentes temáticas, desde el conocimiento de diferentes necesidades educativas como Trastorno de Déficit de Atención con Hiperactividad (TDAH), Trastorno del Espectro Autista (TEA), Altas Capacidades Intelectuales, etc., hasta el uso responsable de las tecnologías y redes sociales, enseñanza de técnicas de estudio, o la gestión de las AMPAS.

Dentro de estos programas tenemos el proyecto Educar en Familia, que oferta formación presencial a los padres dotándolos de las estrategias y herramientas necesarias para la educación de sus hijos. Sus líneas de formación se encuentran representados en tres ejes: la corresponsabilidad educativa o participación de la familia en la vida académica de sus hijos; la convivencia en familia; y, la relación familia y nuevas tecnologías.

Por último, otra de las iniciativas que encontramos en la Red Canaria de Centros para la Participación educativa, que también fomenta la relación familia-escuela, es el 
Programa Hogares Verdes Canarias. El objetivo viene definido en el trabajo conjunto familia-escuela desde la sensibilización hacia el medioambiente. El desarrollo de este proyecto corre a cargo de profesionales que dotan de estrategias a los docentes y a las familias sobre el desarrollo de una participación efectiva entre ambos, y, que lleven a conductas saludables a favor del cambio climático. Además de generar la necesidad de una toma de conciencia responsable sobre el uso responsable y sostenible de los recursos. Todo ello, en un clima de participación y coordinación entre las familias y los centros.

\section{Conclusiones}

La familia y la escuela deben ir de la mano a la hora de educar a los niños, ambos son parte importante y fundamental en este proceso, por eso resulta indispensable reflejar unos objetivos claros y coherentes que favorezcan el desarrollo integral de los menores (Moya, 2018). La responsabilidad socializadora debe recaer tanto en la familia como en la escuela con el fin de evitar disfunciones en la tarea educativa (Parody García, Villalba, del Olmo y Alarcón, 2019). Las escuelas no pueden percibir a los padres como intrusos o confiscadores de su labor educativa (Giró, Mata, Vallespir y Vigo, 2014), todo lo contrario, deben ir de la mano en la educación de los menores. La participación familiar es más efectiva en los centros educativos cuando la comunicación entre sus miembros es bidireccional y activa (Moya, 2018).

Quizás una de las limitaciones que podemos encontrar en la participación familiaescuela es la escasa formación que tienen tanto los docentes como las familias y la dirección de los centros (Martín y Gairín, 2007), de ahí la importancia del apoyo de las Administraciones en dicha formación, tal y como lo está realizando la Comunidad Autónoma Canaria. La Consejería de Educación, Cultura y Deportes del Gobierno de Canarias oferta diferentes cursos formativos para docentes y familias, además de ser pionera en la inclusión de la figura del educador social para diversos fines, como lograr un óptimo desarrollo personal del alumno, participar en el logro de una convivencia cívica y democrática en los centros, o como guía entre los diferentes agentes educativos en el camino de la participación escolar. 
La participación familia-escuela seguirá evolucionando, por ello tendremos que estar atentos a los posibles cambios que se puedan vislumbrar, por ejemplo, ante la llegada de nuevas leyes educativas como podría ser la Ley Orgánica de Modificación de la LOE (LOMLOE).

Este trabajo nos ha permitido conocer el panorama actual de la participación familiaescuela centrándonos especialmente en la experiencia de la Comunidad Autónoma Canaria, de ahí la necesidad de ampliar nuestro estudio a otras comunidades autonómicas para analizar los recursos o estrategias que desarrollan para garantizar la participación entre la institución educativa y la familia.

\section{Bibliografía}

ADOLFI, M. (1991). La familia como sistema relacional. En Terapia Familiar. Un enfoque interaccional. Barcelona: Paidós. 17-35.

BENÍTEZ PÉREZ, M. E. (2017). La familia: Desde lo tradicional a lo discutible. Novedades en población, 13(26), 58-68.

BOLÍVAR, A. (2006). Familia y escuela: dos mundos llamados a trabajar en común. Revista de educación, 339(1), 119-146.

CALVO, M., VERDUGO, M. Á., y AMOR, A. M. (2016). La participación familiar es un requisito imprescindible para una escuela inclusiva. Revista latinoamericana de educación inclusiva, 10(1), 99-113.

CARBONELL, J.; CARBONELL, M. y GONZÁLEZ MARTÍN, N. (2012). Las Familias en el siglo XXI: Una mirada desde el Derecho. Universidad Autónoma del Estado de México, Instituto de investigaciones jurídicas. Serie: Estudios Jurídicos, 205. Coordinadora México. Editorial: Elvia Lucía Flores Ávalos.

CÁRCAMO-VÁSQUEZ, H., y RODRÍGUEZ-GARCÉS, C. (2015). Rol parental educativo: aproximación a las percepciones que poseen los futuros profesores. Educación y Educadores, 1(3), 456-470. DOI:10.5294/edu.2015.18.3.5 
CASTELLANOS, J. (2019). Educación y participación ciudadana: mejorar la docencia universitaria de la mano de los Derechos Humanos. Revista de Educación y Derecho - Education and Law Review, 19, 1-21. DOI: http://dx.doi.org/10.1344/REYD2019.19.29092

COMISIÓN EUROPEA. (2000). Informe europeo sobre la calidad de la educación escolar. Dieciséis indicadores de calidad.

CONSEJERÍA DE EDUCACIÓN Y UNIVERSIDADES (2020). Acceso en: http://www.gobiernodecanarias.org/educacion/

EGIDO, I. (2014), "Marcos normativos de la participación de las familias en los sistemas educativos europeos. Una visión comparada", en Consejo Escolar del Estado (coord.), La participación de las familias en la educación escolar, Madrid: Ministerio de Educación Cultura y Deporte, 35-56.

ENGELS, F (2008) El origen de la familia, la propiedad privada y el Estado. Ed. Colofón S.A. de C.V. México D.F: Reimpresión.

ENTRENA, M. S., y SORIANO, A. (2003). "Escuela de padres”. En E. Gervilla, Educación Familiar. Nuevas relaciones humanas y humanizadoras. Madrid: Narcea. 143-154.

EPSTEIN, J. (2001). School, family, and community partnerships. Preparing educators and improving schools. Boulder, CO: Westview Press.

ESPEJO YASIK, N. (2014). El derecho a la educación en el derecho internacional de los derechos humanos. Revista Pedagogía Universitaria y Didáctica del Derecho, 1 (1), 20-35. DOI: 10.5354/0719-5885.2015.35961

ESTEBAN, J. O. (2014). Educación social y enseñanza: los educadores sociales en los centros educativos, funciones y modelos. Edetania. Estudios y Propuestas Socioeducativas, (45), 11-31. 
GALÁN, D., y CASTILLO, M. (2008). El papel de los educadores sociales en los centros de secundaria. Una propuesta para el debate. Educación social: Revista de intervención socioeducativa, 38, 121-133.

GARCÍA, M. P., HERNÁNDEZ, M. Á., GALIÁN, B. y BELMONTE, M. L. (2020). Docentes, familias y órganos de representación escolar. Estudios Sobre Educación, $38,125-144$.

GARRETA, J. (2016). La participación de las familias en la escuela pública. Las asociaciones de madres y padres del alumnado. Madrid: CEAPA.

GIRÓ, J., MATA, A., VALLESPIR, J. y VIGO, B. (2014). Familias y escuelas: los diferentes discursos sobre la participación. Ehquidad International Welfare Policies and Social Work Journal, (2), 65-90. DOI: https://doi.org/10.15257/ehquidad.2014.0009

GODOY, M. (2018). Familia, modelos y perspectivas. Interacción y perspectiva: Revista de Trabajo Social, 8(1), 115-117.

GONZÁLEZ-PIENDA. J. A., y NÚÑEZ, J. C. (2005). La implicación de los padres y su incidencia en el rendimiento de los hijos. Revista de Psicología y Educación, 1 (1), 115-134.

HERNÁNDEZ, M. A., VIUDEZ, N. y GUERRERO, C. (2015). Percepción de las familias sobre las tutorías en la etapa de Educación Infantil. ENSAYOS, Revista de la Facultad de Educación de Albacete, 30(2), 189- 204.

HUAYAMAVE, K. V., BENAVIDES, B., y SANGACHA, M. (2019). Estudio del rol de los padres de familia en la vida emocional de los hijos. Apuntes Universitarios, $9(2), 61-72$.

LLEVOT, N. y BERNAD, O. (2015). La participación de las familias en la escuela: factores clave. Revista de la Asociación de Sociología de la Educación, 8(1), 57-70. 
MAESTRE, A. B. (2009). Familia y escuela. Los pilares de la educación. Innovación y experiencias educativas, 14, 1-11.

MARÍN, M. P., QUINTERO, P., y RIVERA GÓMEZ, S. C. (2019). Influencia de las relaciones familiares en la primera infancia. Poiésis, (36), 164-183.

MARINA, J. A. (2004). Aprender a vivir. Barcelona: Ariel.

MARTÍN, M. y GAIRÍN, J. (2007). La participación de las familias en la educación: un tema por resolver. Bordón. Revista de pedagogía, 59 (1), 113-151.

MARTÍN, J. C. y MESA J. L. (2015). Familia y escuela en la sociedad de la comunicación. Las Palmas: Universidad de Las Palmas de Gran Canaria.

MARTÍN-MORENO, Q. (2000): Bancos de talento. Participación de la comunidad en los centros docentes. Madrid: Sanz y Torres.

MARTÍNEZ, A. (ed.). (2005). La familia como ámbito educativo. Ediciones Rialp.

MENACHO, S. (2013). El educador social y la escuela. Justificación de la necesidad de la Educación Social en la escuela. RES. Revista de Educación Social, 16, 1-16.

MESTRES, L. (2012). Cómo mejorar la participación de las familias en la escuela. Monográfico Familia y Escuela, 247.

MORENO-ACERO, I. D., LEYVA-TOWNSED, P., Y PARRA-MORENO, C. (2019). La Familia, primer ámbito de educación cívica. Civilizar: Ciencias Sociales y Humanas, 19(37), 1-24.

MOYA, V. V. (2018). La interacción de la familia en la escuela. Padres y profesores: nuevas vías de comunicación. Hekademos: revista educativa digital, (25), 62-72.

OLIVA, E. (2013). El Divorcio incausado en México. México: Moreno Editores.

ORTÍN, L. R. (2012). El educador social: ética y práctica profesional. Pedagogía social: revista interuniversitaria, (19), 51-63. 
ORTIZ CERMEÑO, E. (2011). “Comunidad Educativa: ámbito de colaboración entre la familia y la escuela”. En, J. J. Maquilón Sánchez, A. B. Mirete Ruiz, A. Escarbajal Frutos y A. Ma Giménez Gualdo (coords.), Cambios educativos y formativos para el desarrollo humano y sostenible, 71-80.

PARODY GARCÍA, L., VILLALBA, M. J., DEL OLMO, M. J., y ALARCÓN, E. I. (2019). El desafío educativo del siglo XXI: relevancia de la cooperación entre familia y escuela. Espiral. Cuadernos del Profesorado, 12(24), 19-29.

PLIEGO, F. (2013). Tipos de familia y bienestar de niños y adultos. Instituto de Investigaciones Sociales: México.

QUINTERO, A. M. (2007). Diccionario especializado en familia y género ( $1^{\mathrm{a}} \mathrm{ed}$.). Buenos Aires: Lumen, 59-67.

REGO, M., MOLEDO, M. L., y CAAMAÑO, D. P. (2019). La Mejora de la Participación e Implicación de las Familias en la Escuela: un Programa en Acción. Revista Electrónica Interuniversitaria de Formación del Profesorado, 22(3), 93-107.

RODRIGO, M. J. y PALACIOS, J. (1998). "Marcos conceptuales en el análisis evolutivo-educativo de la familia", en M. J. Rodrigo y J. Palacios (Coord.) Familia y desarrollo humano. Madrid: Alianza.

RODRIGO, M. J.; MÁIQUEZ, M. L.; MARTÍN, J.C. y BYRNE. S. (2008). Preservación familiar: un enfoque positivo para la intervención con familias. Madrid: Pirámide.

SAN FABIÁN, J. L. (dir.) (1997). La experiencia participativa de los estudiantes en el ámbito escolar. Madrid: MEC/CIDE.

SAN FABIÁN, J. L. (2006). "Participación de las familias y Acción Tutorial: dos puertas para el cambio", en García, A (coord.), Participación de las familias en la vida escolar: acciones y estrategias. Ministerio de Educación y Ciencia: Secretaria General de Educación. 131-148. 
SANTOS GUERRA, M. A. (1997): El crisol de la participación. Investigación sobre la participación en Consejos Escolares de centros. Archidona: Aljibe.

SEGOVIA, J. D., TITOS, M. A., y MARTOS, L. D. (2016). Colaboración familiaescuela en España: retos y realidades. REXE-Revista de Estudios y Experiencias en Educación, 9(18), 111-133

SOSA, J. A. (2009). Evolución de la relación familia-escuela. Tendencias Pedagógicas, 14, 251-265.

TORRES, M. (2013). El capital social en las asociaciones de madres y padres: Formación, desarrollo e institucionalización. Granada: Universidad de Granada.

UNESCO. (1994). Declaración de Salamanca y marco de acción ante las necesidades especiales.

VICENTE, M. A. G., PRAdOS, M. Á. H., SANZ, M. P. G., Y MARTÍNEZ, J. P. (2017). Tejiendo puentes entre la escuela y la familia. El papel del profesorado. Bordón. Revista de pedagogía, 69(2), 41-57.

VILREALES, P. T., ÁLVAREZ, F. V., y CERÓN, G. M. (2004). ¿Qué hacen y qué pueden hacer las AMPAs? Cuadernos de pedagogía, (333), 53-57.

VISHER, E. y VISHER, J. (1988). Old Loyalties, new ties: Therapeutic strategies with Stepfamilies. Brunner/Mazel, N. York.

WARREN, M. R. (2005). Communities and schools: A new view of urban education reform, Harvard Educational Review, 75 (2), 133-173. 